und Metaanalysen beziehen sich nur auf die Kurzzeittherapie“, erläuterte Professor Christopher Baethge von der Klinik und Poliklinik für Psychiatrie und Psychotherapie vom Universitätsklinikum Köln.

In der jetzt vorgelegten Metaanalyse aus 29 Studien, in denen die Suizidrate angegeben wurde, konnten die Daten von 4.016 Patienten mit einer über drei Monate dauernden Antidepressivathera- pie mit denen von 2.918 Patienten unter Placebo verglichen werden. Insgesamt fand sich mit nur acht Suiziden und 14 Suizidversuchen eine sehr niedrige Ereignisrate. Dies entspricht 1,45 Suiziden und 2,76 Suizidversuchen pro $1.000 \mathrm{~Pa}$ tientenjahre. Im Vergleich zu Placebo war die Suizidrate unter dem Antidepressivum erhöht (Suizide: 2,18 vs. 0,43/1.000 Patientenjahre, $\mathrm{p}=0,102$, Suizidversuche 4,34 vs. $0,48 / 1.000$ Patien- tenjahre, $\mathrm{p}=0,007)$. „Somit gibt es keine Evidenz für eine suizidpräventive Wirkung einer länger als drei Monate durchgeführten Therapie mit einem Antidepressivum", so Baethge.

Dr. med. Peter Stiefelhagen

Symposium "Antidepressiva richtig einsetzen Praktische und wissenschaftliche Erkenntnisse aus drei unpublizierten systematischen Metaanalysen", DGPPN-Kongress, Berlin,

25. -28.11 .2015$

\title{
Wer tut so was? - Wenn die Liebe tödlich endet
}

\section{Der Intimizid - die Tötung des Intimpartners - ist das dramatische Ende einer festen Beziehung, die einmal mit Liebe und Hoffnung begann. Die Vorgeschichte bis zu dieser totalen Katastrophe reicht meist jahrelang oft auch jahrzehntelang zurück.}

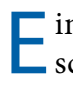
ine Kategorisierung der Intimizide ist schwierig, jeder Einzelfall muss multidimensional betrachtet und ausgelotet werden, erklärte Professor Andreas Marneros, Halle, der als forensischer Psychiater jahrzehntelange Erfahrung mit der Begutachtung solcher Fälle hat. Die Katastrophe hat immer zwei Protagonisten, betonte er. Die Interaktionssysteme, die emotionalen und psychischen Prozesse und die mögliche Psychopathologie beider Partner müssen exploriert werden. Auch der Tathergang selbst ist wichtig und keine Einmischung in die Arbeit des Staatsanwalts, bekräftigte Marneros: Die Intentionali-

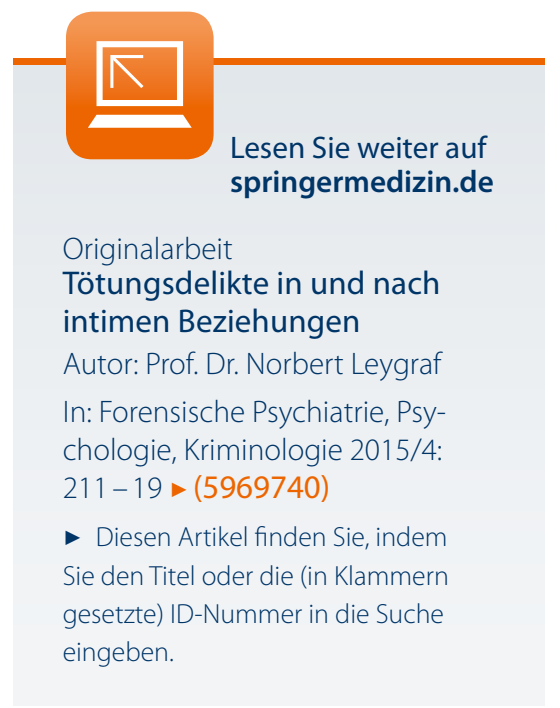

tät und die Peristase wenige Minuten und Stunden vor der eigentlichen Tat sind wichtig auch für die Begutachtung durch den forensischen Psychiater.

\section{Selbstdefinition tief erschüttert}

Etwa sieben von zehn Intimiziden geschehen in einer etablierten Partnerschaft, oft aus einer jahrzehntelang aufgebauten Erschütterung der Selbstdefinition des Täters heraus. „Unser aller Selbstdefinition braucht die Reflexion der anderen", sagte Marneros. Wenn dies in der Beziehung dauerhaft versagt bleibe und auch extern keine Quelle zur Stützung der Selbstdefinition bestehe, werde die Selbstdefinition erschüttert. Faktoren, die zu einer solche Entwicklung beitragen sind seinen Ausführungen nach

_ein ungleicher Grad der Fixierung auf die Beziehung,

_eine deutliche teleologische Asymmetrie - die Partner investieren unterschiedlich viel in die Beziehung, - ein geringer Grad der interaktionalen Komplettheit - die Partner ergänzen sich nicht gut - und

—eine geringe Verfügbarkeit von Alternativressourcen.

Dies alles ist der Nährboden für eine zunehmende Destabilisierung der Beziehung mit Zweifeln des zukünftigen Täters an sich selbst und am anderen. Es kann zur konzeptionellen Desorientie-

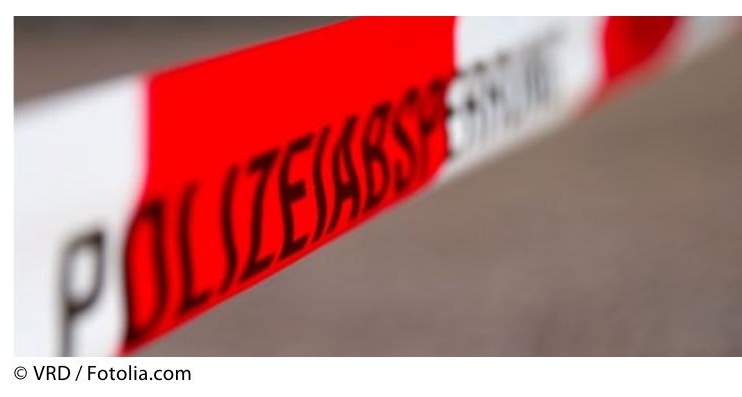

rung des Täters kommen und schließlich zur "finalen Bankrottreaktion“, wie Marneros es nannte: Zum Intimizid als Prototyp der Affekttaten an einem relevanten Anderen, bei dem alle Hemmungs- und Bewältigungsmechanismen zusammenbrechen.

Täter sind häufig sthenisch unterlegene, chronisch gekränkte und/oder lebensbankrotte Intimpartner. Der Intimizid richtet sich gegen den die Lebensordnung Zerstörenden (z. B. weil der die Trennung will), kann aber auch eine autoprotektive Reaktion sein oder ein Emanzipations- und Befreiungsausbruch.

Besonders tragisch ist, wenn der Intimizid mit Ersatzopfern erfolgt: Die geliebten gemeinsamen Kinder werden getötet, um den Intimpartner zu bestrafen und ihm lebenslange Leiden beizubringen. Das kann insbesondere eintreten, wenn der Täter einen malignen Narzissmus aufweist: eine narzisstische Persönlichkeitsstörung gepaart mit antisozialem Verhalten, einer Ich-syntonen Aggression oder Sadismus gegen andere und eine ausgeprägt paranoide Haltung.

Friederike Klein

Meet the Expert „Intimizid“, DGPPN-Kongress, Berlin, 27.11.2015 